\title{
Analytische Mikrosysteme für medizinische Anwendungen
}

\author{
Gerald A. Urban, M. Bergmann, Albert-Ludwigs-Universität Freiburg, IMTEK, Georges Koehler Allee \\ 103, 79110 Freiburg, Deutschland \\ Urban@imtek.de
}

\begin{abstract}
Zusammenfassung
Biosensoren, vor allem Glukose-Biosensoren, gehören zu einem Sensor-Marktsegment mit einem Umsatz von ca. 10 Milliarden \$ pro Jahr und schlagen damit alle anderen Einzel-Sensor-Produkte. Solche Sensoren werden primär als Einwegsensoren für das Diabetes-Management verwendet. Zukünftige Entwicklungen werden eine verstärkte Nachfrage nach Point-of-Care-Geräten und insbesondere Monitoring-Geräten zeigen, welches die Entwicklung von Lab-on-Chip-Systemen zur Verbesserung der Diagnostik in der personalisierten Medizin erfordert.

Die personalisierte Medizin möchte eine Kohorten-basierte Patientenbehandlung etablieren, wozu Mikrobiosensoren zur Überwachung von metabolischen Parametern benötigt werden. Die nächste Generation von Biosensoren sollte für längere Zeitskalen im Körper eines Patienten arbeiten und muss daher biokompatibel werden. Die Forschungen über Biokompatibilität begannen in den sechziger Jahren des letzten Jahrhunderts und behandeln Fragestellungen, welche immer noch nicht gelöst ist. Es wird der Stand der Technik der Biosensoren präsentiert und danach die Herausforderungen, welche sich mit Fragen der Biokompatibilität auftauchen diskutiert. Ein neuer Weg zur Biokompatibilisierung von Implantaten wird vorgestellt, der auf dem Konzept der In-situBiologisierung basiert. Dieses Konzept wurde mittels in-vitro und in-vivo Versuche evaluiert und wird zur Diskussion gestellt.
\end{abstract}

Keywords: Biosensoren,Bioanalytik,Mikrosystemtechnik in-vivo Sensorik,Biokompatibilität

\section{Einleitung}

Die Fortschritte der Mikrosystemtechnik haben die Erwartung geweckt, einen umfassenden Einblick in die metabolische Situation von Patienten $\mathrm{zu}$ erhalten. Dies ist der Grundgedanke der sogenannten personalisierten Medizin, welches die Diagnostik und Therapie von Patienten Kohorten-basiert durchführen möchte. Gegenwärtig werden alle akuten Interaktionsuntersuchungen von metabolischen Parametern unter Verwendung von Point-of-Care (POC)-Analysatoren durchgeführt, die mit miniaturisierten Sensorarrays arbeiten. Um diese Vielzahl von Stoffwechselparametern präzise und selektiv in einer komplexen Analytmatrix, wie zum Beispiel Blut, nachzuweisen, müssen Biosensoren eingesetzt werden. Hier ist als erstes Beispiel der miniaturisierte
Glukosesensor zu nennen, der eine genauere Insulineinstellung von Diabetikern ermöglicht.

Solche Mikrobiosensoren können auch
integriert werden, um verschiedene
Blutparameter simultan zu analysieren. In
weiterer Folge können diese in einer
Mikrofluidik-basierten Monitoring-Analyse-
einheit zur kontinuierlichen Messung von
Körperflüssigkeiten implementiert werden.

\section{Mikrobiosensoren}

Die Messung von Stoffwechselparametern durch Mikrosensoren in vitro und in vivo können durch Immobilisierung von Enzymen auf modifizierten Mikroelektroden als Bio- oder Chemosensoren realisiert werden. Dabei ermöglichen diese Enzyme mit ihrer sehr spezifischen Analyt-Wechselwirkung die Herstellung von selektiven hochminiaturisierbaren elektrochemischen Sensoren, wobei der gemessene Strom 
proportional zur Analytkonzentration ist. Das prominenteste Beispiel ist der Glukosesensor, welcher ein Enzym auf einer elektrochemischen Elektrode adsorbiert hat, eine einfache Mikrofluidik aufweist und damit eines der ersten Lab-on.-Chipsysteme darstellt welches Millionenfach verkauft wurde [1].

Eine Weiterentwicklung stellen integrierte Biosensoren in sogenannten Point of Care Analysatoren dar, die in eine mikrofluidischen Cartridge implementiert werden [2].

Als nächsten Schritt kann man die Sensorik noch weiter an den Patienten mittels sogenannter kontinuierlich arbeitenden Biosensoren bringen. Die am meisten untersuchten In-vivo-Sensoren sind sicherlich kontinuierliche Glukoseüberwachungssysteme (CGM), welche Patienten mit Stoffwechselstörungen, z. B. Diabetes eine lückenlose sicherstellen sollen [3].

\section{Kontinuierlich arbeitende implantierbare} Mikrobiosensorsysteme

Um eine kontinuierlich arbeitende Analyseeinheit für Patienteneinsatz zu realisieren, müssen sogenannte Lab-onChipsysteme eingesetzt werden. Dabei müssen integrierte und miniaturisierte Mikrobiosensoren in eine Mikrofluidik implementiert werden. Hier konnten neuerdings integrierte Biosensoren in Kombination mit einer Mikrodialyse am Patienten in der Intensivstation Eingang finden, um kontinuierliche Überwachung der Glukoseund Laktatwerte am Krankenbett zu ermöglichen [4].

Eine kontinuierliche implantierte Überwachung von Glukose über einen längeren Zeitraum bis zu einer Woche im Heimbereich ist möglich, allerdings mit etwas eingeschränkter Genauigkeit [5].

Das Endziel ist aber der Einsatz von Mikrobiosensoren in künstlichen oder hybriden Organsystemen. Hier ist allerdings, obwohl seit Jahrzehnten Forschungen betrieben werden, noch kein Durchbruch gelungen [6].

Hierbei müssen Schwierigkeiten, die vom Sensorprinzip abgeleitet werden, können von Gewebe-Sensor Abstoßungssreaktionen unterschieden werden.

Mit den Abstoßungsreaktionen des Körpers Problemen befasst sich die Biokompatibilitätsforschung. Während über technische Realisierung von zuverlässigen und hochminiaturisierten in-vivo tauglichen
Sensoren hinreichend berichtet worden ist (Abb. 1) und Probleme, welche mit Sensorkontamination einhergehen durch spezielle Elektroden-Modifikationen und Membransystemen minimiert werden können [7], ist die Biokompatibilitätsforschung noch nicht zu zufriedenstellenden Ergebnissen gekommen [8].

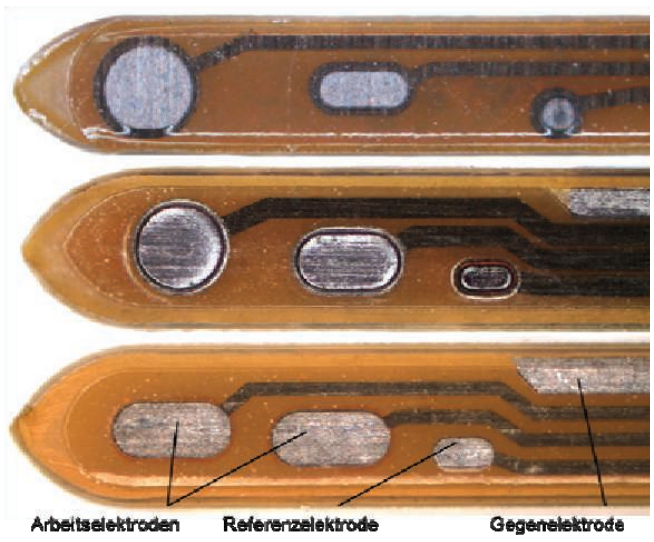

Abb. 1: Verschiedene Varianten von in-vivo Biosensorarrays

\section{Biokompatibilität}

Bei der Entwicklung von biokompatiblen Materialien für biomedizinische Anwendungen ist eine maßgeschneiderte Fremdkörperreaktion ein wichtiges Thema. Die Heilung von umgebendem Gewebe beeinträchtigt oft die Funktion eines Implantats. Ereignisse wie Proteinablagerung, Fremdkörperreaktion, Infektionen und die unerwünschte Verkapselung des Implantats sind die Hauptursachen für das Versagen implantierter Geräte [9-12].

Eine neue Methode verwendet biokompatible Nanofilme, abgeschieden mittels eines Plasmapolymerisationsverfahrens unter Verwendung einer Niederdruck-Magnetronverstärkten $15 \mathrm{kHz}-\mathrm{Glimmentladung.} \mathrm{Es} \mathrm{wurde}$ eine neuartige Anlage konzipiert, in der sowohl Parylen- wie auch Plasmapolymerisation in einer geschlossenen Vakuumbeschichtungsanlage aufgebracht werde konnte (Abb.2). 


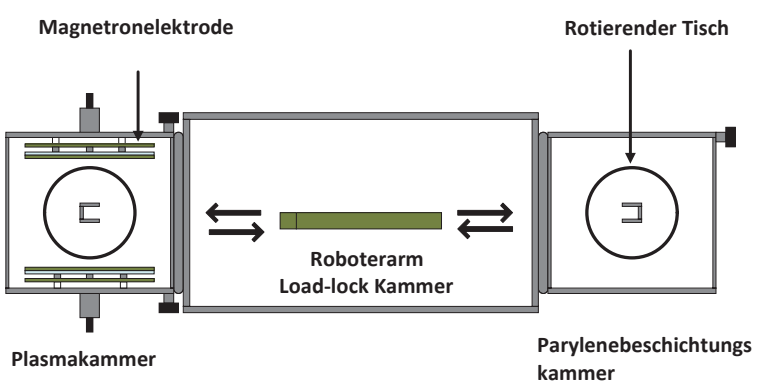

$\begin{array}{llr}\text { Abb. 2: } & \text { Schema einer integrierten } \\ & \text { Plasmapolymerisations- } & \text { und } \\ & \text { Parylenebeschichtungsanlage } & \end{array}$

Dieses Verfahren ermöglicht die genaue Kontrolle der Filmeigenschaften und des physiko-chemischen Verhaltens. Der resultierende Kohlenwasserstofffilm hat eine Dicke von nur wenigen Nanometern und beeinflusst somit nicht die inhärenten Eigenschaften des Substratmaterials. [13], [14] Neben verschiedenen oberflächenanalytischen Methoden wurde die Interaktion mit verschiedenen biologischen Probenmaterialien getestet.

Verschiedene In-vivo-Sensorkörper in Kontakt mit Blut und Weichteilen wurden mit Nanofilmen beschichtet. Die native Sekundärstruktur von Proteinen hielt die implantierten Sensoren frei von jeglicher Verkapselung durch eine PlasmaNanofilmbeschichtung [13]. Dieses Verfahren wird als in-situ Biologisierung definiert.

Die Messergebnisse der Adsorption von Blutproteinen zeigten, dass diese Nanofilme bereits sehr biokompatibel sind. Die in-vivoPrüfung von beschichteten Sensoren wies darauf hin, dass sie frei von jeglicher Verkapselung waren. Diese neuartigen Beschichtungen öffnen damit die Tür für viele neue Anwendungen auf dem Gebiet der Biomedizin.

\section{Resüme}

Die Mikrosystemtechnik kann aktuell tatsächlich schon kommerziell realisierte Biosensorarryas für die personalisierte Medizin anbieten, welche schon Stand der Technik sind. Bei kontinuierlich arbeitenden und insbesondere implantierbaren Biosensoren herrscht aber weiterhin noch großer Forschungsbedarf. Neue Methoden können beitragen, das Biokompatibilitätsproblem bei bestimmten analytischen Fragestellungen zu verringern. Der Weg zu universell einsetzbaren und langzeitstabilen implantierbaren Biosensoren ist aber immer noch weit.

\section{Literaturnachweis}

[1] Urban G, Micro- and Nanobiosensors - State of the Art and Trends, Meas Sci Technol, Band: 20, Nummer: 1, (2008), 012001.

[2] http://www.novabio.us/prime/

[3] David Rodbard, Diabetes Technol Ther. Feb 1; 18(Suppl 2), (2016) S2-3-S2-13.

[4] https://www.maquet.com/de/produkte/eirus/ 30.10.2017

[5] J. Kropff, D. Bruttomesso, W. Doll, A. Farret, S. Galasso, Y. M. Luijf, J. K. Mader, J. Place, F. Boscari, T. R. Pieber, E. Renard and J. H. DeVries, Diabetes, Obesity and Metabolism Volume 17, Issue 4 (2015), 343-349

[6] M. Shichiri et al.: Artificial Systems for Insulin delivery. Ed. By Brunetti et al. (1983), 445-455

[7] Weltin A, Kieninger J, Urban G, Anal Bioanal Chem, Band: 408, (2016), 4503 - 4521

[8] B.D. Ratner, Journal of Cardiovascular Translational Research, 4, 5, (2011), 523-527

[9] H. Yasuda, Q. S. Yu, and M. Chen, Progress in Organic Coatings,. 41(4) 27 (2001) 3-279.

[10] M.B. Gorbet, M.V. Sefton, Biomaterials, 25, 26, (2004), 5681-703

[11] T.A. Horbett, Cardiovascular Pathology, Chapter 13, 2, 3, (1993), 137-148

[12] J. C. Barrese, et al., Journal of Neural Engineering, 10, 6, (2013), 066014

[13] M. Bergmann, et al., Plasma Processes and Polymers, 10, 11, (2013), 970-977

[14] L. Ledernez, PhD. Thesis, (2011), University Freiburg - Imtek. 